OPEN ACCESS

Edited by:

Amy Lovett-Racke,

The Ohio State University,

United States

Reviewed by:

Bruce V Taylor,

University of Tasmania, Australia

Bonaventura Casanova,

University and Polytechnic Hospital of La Fe, Spain

*Correspondence:

Laura Espino-Paisán

lauraep80@gmail.com

tThese authors have contributed equally to this work

Specialty section: This article was submitted to

Multiple Sclerosis

and Neuroimmunology,

a section of the journal

Frontiers in Immunology

Received: 21 January 2020

Accepted: 06 April 2020

Published: 05 May 2020

Citation: Espino-Paisán L,

Agudo-Jiménez T, Rosales-Martínez I, López-Cotarelo $P$

García-Martínez MÁ

Domínguez-Mozo MI, Pérez-Pérez S, Dieli-Crimi R, Comabella M, Urcelay E

and Álvarez-Lafuente $R$ (2020) A Polymorphism Within the MBP Gene Is Associated With a Higher Relapse

Number in Male Patients of Multiple Sclerosis. Front. Immunol. 11:771. doi: 10.3389/fimmu.2020.00771

\section{A Polymorphism Within the MBP Gene Is Associated With a Higher Relapse Number in Male Patients of Multiple Sclerosis}

\author{
Laura Espino-Paisán 1*, Teresa Agudo-Jiménez', Isabel Rosales-Martínez', \\ Pilar López-Cotarelo', María Ángel García-Martínez², \\ María Inmaculada Domínguez-Mozo², Silvia Pérez-Pérez², Romina Dieli-Crimi ${ }^{3}$, \\ Manuel Comabella ${ }^{4}$, Elena Urcelay ${ }^{1 \dagger}$ and Roberto Álvarez-Lafuente ${ }^{2 \dagger}$
}

\begin{abstract}
' Laboratorio de Investigación en Genética de Enfermedades Complejas, Instituto de Investigación Sanitaria del Hospital Clínico San Carlos (IdISSC), Red Española de Esclerosis Múltiple (REEM), Madrid, Spain, ${ }^{2}$ Grupo de Investigación de Factores Ambientales en Enfermedades Degenerativas, Instituto de Investigación Sanitaria del Hospital Clínico San Carlos (IdISSC), Red Española de Esclerosis Múltiple (REEM), Madrid, Spain, ${ }^{3}$ Servicio de Inmunología, Hospital Universitari Vall d'Hebron (HUVH), Diagnostic Immunology, Vall d'Hebron Research Institute (VHIR), Barcelona, Spain, ${ }^{4}$ Servei de Neurologia-Neuroimmunologia, Centre d'Esclerosi Múltiple de Catalunya (Cemcat), Institut de Recerca Vall d'Hebron (VHIR), Hospital Universitari Vall d'Hebron, Universitat Autònoma de Barcelona, Red Española de Esclerosis Múltiple (REEM), Barcelona, Spain
\end{abstract}

Myelin basic protein (MBP) is thought to be one of the key autoantigens in multiple sclerosis (MS) development. A recent study described the association of the single nucleotide polymorphism (SNP) rs12959006, within the MBP gene, with a higher risk of relapse and worse prognosis. We aim at studying potential associations of this SNP to MS in an independent population. Clinical data of the first 5 years of the disease were collected retrospectively from 291 MS confirmed patients. MBP polymorphism rs12959006 was genotyped in all patients. Associations with EDSS, number of relapses and serology for Herpesvirus 6 (HHV-6) and Epstein Barr (EBV) viruses were studied. Lymphocyte activation measured by CD69 expression was also analyzed according to sex and rs12959006 genotype. The rs12959006 polymorphism contributed significantly to a higher number of relapses at 5 years after onset only in male patients (rs12959006*TT $\beta=0.74$ [0.36-1.09]; $p=7 \times 10^{-5}$ ). Titers of anti-HHV6 IgG antibodies showed also a mild association with relapses, both in male and female patients $\left(\beta=0.01\right.$ [0.01-0.02]; $\left.p=3.7 \times 10^{-8}\right)$. Both the genetic variation in $M B P$ and HHV-6 infection aid in predicting a higher number of relapses during the first years of MS. The association described in MBP rs12959006*T is exclusive to male patients.

Keywords: multiple sclerosis, SNP, HHV-6, myelin basic protein, relapse, sex differences, genetic marker

\section{INTRODUCTION}

Multiple sclerosis (MS) is a chronic inflammatory and demyelinating disease affecting the central nervous system (CNS) and is one of the primary neurological causes of physical disability in young adults (1). The underlying mechanism seems to be an autoimmune response to unidentified antigens in the CNS. One of these proposed antigens is the myelin basic protein (MBP), 
the second most abundant component of the myelin sheath, and a protein that plays an important role in the myelination process. Interestingly, MBP is part of the peptide cocktail used for inducing experimental autoimmune encephalomyelitis (EAE) in mice, along with proteolipid protein (PLP) and myelin olygodendrocyte glycoprotein (MOG) (2). Although MBP has not been proved to be the key autoantigen in MS, MBP-specific autoreactive T-cells have been found in blood of MS patients at a higher rate than in healthy individuals (3).

Several studies have focused on finding new markers for MS diagnosis and prognosis that are cost-effective, reliable, easy to implement and as minimally invasive as possible. Many candidates have been singled out in individual studies (4), but a necessity exists to replicate these findings in independent cohorts to ascertain the validity and reproducibility of these new potential biomarkers. Genetic markers are especially interesting due to their easy determination, stability through time and accessibility of the sample. Genome-wide association studies (GWAS) have identified more than 200 polymorphisms associated with susceptibility to MS (5). Additional studies with carefully selected patients are warranted to find genetic markers that can be used as predictive and prognostic biomarkers for MS.

A recent study pointed to polymorphism rs12959006 within the $M B P$ gene as a risk modifier of conversion to definite MS and progression of the disease (6). The authors studied genetic markers in a prospective cohort after a first demyelinating event. They found that the rs12959006 minor allele T was associated with a higher risk of conversion to MS, and a greater hazard of relapse $(\mathrm{HR}=1.74[1.19-2.56] ; p=0.005)$ and annualized disability progression $(\beta=0.18[0.06-0.30] ; p=0.004)$. Moreover, in this study, the risk genotype carrying the $\mathrm{T}$ allele showed an interaction with baseline titers of IgG antibodies against Human Herpesvirus 6 (HHV-6). We aimed at studying this polymorphism and its putative interaction with viral antibodies against viruses relevant to MS in an independent cohort of MS-confirmed patients.

\section{MATERIALS AND METHODS}

\section{Patients}

A total of 291 patients $(66.7 \%$ female $)$ were included in the study. Patients were diagnosed with relapsing remitting multiple sclerosis (RRMS) between 1991 and 2014 according to the Poser or McDonald criteria (7-10), and followed in the Multiple Sclerosis Unit at Hospital Clinico San Carlos (Madrid, Spain). Mean age at onset was $29.40 \pm 7.78$ years. Medical records of each patient were reviewed and data from the first 5 years after a first demyelinating event were extracted. Patients were selected based on two criteria: the patient was followed in the unit from MS onset and clinical data such as relapse and EDSS for the first 5 years of the disease were available. Due to a slightly higher availability of relapse data, this variable was chosen as inclusion criterion over EDSS. Written informed consent was obtained from each patient and the study was approved by the Ethics Committee of Hospital Clínico San Carlos.
Clinical data collected included EDSS at 2 and 5 years after onset, relapse in the 30 days prior to EDSS measurement, number of relapses at 2 and 5 years after onset, age at disease onset and disease modifying treatments employed during the evaluated period. Titer of anti-HHV-6 (IgG and IgM) and antiEBV (EBNA-1 and VCA) antibodies, against two viral agents related to MS that have been previously associated to disease course, were also determined.

Seventy-one percent of patients received disease modifying treatments during the 5 year follow-up, with $28.3 \%$ (82 patients) remaining without treatment; among the treated patients, $85.6 \%$ (178) received only first line treatments (interferon- $\beta$ and/or glatiramer acetate) during the period of study. The remaining $14.4 \%$ (30) patients received a combination of first line and second line treatments (natalizumab or mitoxantrone, among others). There were no statistically significant differences in the groups studied concerning the distribution of treatment options (no treatment, first line therapies, combined use of first line and second line therapies). To adjust for treatment effect, all multivariable regression models included two variables: "DMT use," defined as no treatment versus use of any disease modifying treatment in the first 5 years of the disease, and "Onset before 2002," to account for the reduced treatment options and different therapy approaches before the availability of natalizumab and other second line treatments. The year 2002 was selected because a Spanish patient with onset of disease in that year would have the option to be treated with natalizumab at least 1 year in the 5 year follow-up considered for the study.

Viral antibody data were available for a maximum of 227 patients. In several cases, viral antibody measurement was performed after the 5-year period considered for evaluation (mean duration of MS until antibody determination was $7.47 \pm 4.78$ years). Statistical tests were performed to compare antibody titers from patients evaluated close to diagnosis and those with a longer evolution of disease and no differences were found. Despite these results, antibody titers were always adjusted by duration of disease until measurement when used in regression studies. Viral antibodies were determined in serum samples with ELISA commercial kits: ELISA-VIDATEST antiHHV-6 IgG and IgM (Vidia), Captia EBNA-1 IgG and VCA-EBV IgG (Trinity Biotech), according to the manufacturer's protocols.

\section{Genotyping}

Patients were genotyped for the $M B P$ polymorphism rs12959006*C $>$ T with Taqman assay C__3079229_20 in a $7900 \mathrm{HT}$ Fast Real-Time PCR system (Life Technologies, United States). The genotyping success rate was $100 \%$.

\section{In vitro Activation Analysis and Flow Cytometry}

Peripheral blood mononuclear cells (PBMCs) from 75 patients were selected and divided in 4 groups according to sex and genotype at rs12959006 (approximately 19 patients per group). Due to the low frequency of the minor homozygote, only major homozygote and heterozygote patients were included. Treatment effect was accounted for by including patients with the same 
treatment in every group. Samples were cultured in RPMI supplemented with $10 \%$ FBS, $1 \%$ penicillin-streptomycin and 1\% L-glutamine (Sigma-Aldrich, Bremen, Germany), without stimulus or treated with $5 \mu \mathrm{g} / \mathrm{ml}$ Concanavalin A (Con A, Sigma-Aldrich, Bremen, Germany). Afterward, they were kept at $37^{\circ} \mathrm{C}$ and $5 \% \mathrm{CO}_{2}$ for $24 \mathrm{~h}$. Cells were stained with anti-CD69-FITC, anti-CD3-PE, and 7-AAD to exclude nonviable cells (Biolegend, San Diego, CA, United States). Samples were processed and analyzed in a Cytomics FC500 flow cytometer with Kaluza 2.0 software (Beckman Coulter, Brea, CA, United States) and percentage of $\mathrm{CD}^{+} 9^{+}$cells and median fluorescence intensity (MFI) of $\mathrm{CD}^{+} 9^{+}$cells in $\mathrm{CD}^{+}$and $\mathrm{CD}^{-}$ populations were measured. Final values were calculated by extracting the basal CD69 measures (without stimulus) to the samples treated with Con A.

\section{Statistical Analysis}

EDSS and number of relapses at 5 years after onset were evaluated. Data at 2 years after onset were used to confirm the obtained results at a different time point. For multivariable analysis, all variables were analyzed in a correlation matrix with Pearson and Spearman coefficients. Relevant clinical features that showed significant correlation with the dependent variable were thus selected, and models were built to check for joint contribution and to adjust for potential confounding variables. Influence of rs12959006 in EDSS was analyzed with linear regression; influence in relapses, with Poisson regression. Linear regression analyses were performed with SPSS v15.0.1, Poisson regression analyses with RStudio v1.2.5019, and graphical representations were carried out with GraphPad Prism V5.01.

\section{RESULTS}

All participants were genotyped for rs12959006 (minor allele $\mathrm{T}$ frequency $=0.18$ ). Allele frequencies were concordant with those reported for the Iberian population in the 1000 Genomes database.

We aimed at studying the contribution of rs12959006 genotype in relapse and EDSS at 5 years after onset. For relapse, we analyzed the number of relapses by genotype using Poisson regression. The rs12959006*TT genotype showed a statistically significant association with higher number of relapses $(\beta=0.32[0.07-0.55] ; p=0.008)$ in all patients. Surprisingly, when stratified by sex, both rs12959006 genotypes carrying the minor allele $\mathrm{T}$ showed association only in male patients (Table 1).

We investigated other variables that correlated significantly with relapse to build a multivariable model. Out of the four viral serology studies (IgG and IgM for HHV-6 virus and EBNA-1 and VCA for EBV) only IgG anti-HHV-6 antibodies showed correlation and were included in further statistical analyses, corrected by duration of disease until antibody measurement. Use of DMT during the 5 years evaluated was also included, and adjusted for "Onset before 2002" (as detailed in the Methods section), to account for treatment effect. When analyses were re-run, the rs $12959006^{*}$ TT genotype showed a slightly stronger statistical association with relapse at 5 years after onset in the general population $(\beta=0.36$ [0.08-0.63]; $p=0.009$ ) (Table 2). In patients stratified by sex, both rs $12959006^{*} \mathrm{CT}$ and TT genotypes showed statistically significant association with a higher number of relapses only in male patients (Table 2), with a stronger effect of the rs12959006 minor homozygote $(\beta=0.74$ [0.36-1.09]; $\left.p=7 \times 10^{-5}\right)$.

Interestingly, titers of IgG anti-HHV6 antibodies were independently associated with higher number of relapses in both male and female MS patients (Table 2).

We attempted at replicating these observations with relapse data of the first 2 years of the disease. Although the effect of rs12959006 was weaker, when evaluating carriers of rs $12959006^{*} \mathrm{~T}$ it was observed that the presence of the minor allele in male patients was associated with a higher number of relapses $(\beta=0.30$ [0.02-0.57]; $p=0.03$ ) while it showed no effect in female patients $(\beta=-0.05[-0.12$ to 0.80$] ; p=0.78)$ or in the general population $(\beta=0.11[-0.07$ to 0.68$] ; p=0.23)$.

For EDSS we built multivariable linear regression models with rs12959006 and the other selected variables and no statistically significant contributions were found.

To evaluate whether potential alterations in immune cells correlated with the presence of the $\mathrm{rs} 12959006^{*} \mathrm{~T}$ allele, we analyzed activation in PBMCs of MS patients through stimulation

TABLE 1 | Poisson regression to test for influence of rs12959006 genotype in number of relapses at 5 years after MS onset.

\begin{tabular}{|c|c|c|c|}
\hline & Variables included & $\beta(95 \% \mathrm{Cl})$ & $p$ \\
\hline MS overall & rs12959006*CC & Reference & Reference \\
\hline \multirow[t]{2}{*}{$N=291$} & rs12959006*CT & $0.07(-0.04$ to 0.19$)$ & 0.24 \\
\hline & rs12959006*TT & $0.32(0.07-0.55)$ & 0.008 \\
\hline MS males & rs12959006*CC & Reference & Reference \\
\hline \multirow[t]{2}{*}{$N=97$} & rs12959006*CT & $0.21(0.00-0.41)$ & 0.03 \\
\hline & rs12959006*TT & $0.68(0.35-0.98)$ & $1.8 \times 10^{-5}$ \\
\hline MS females & rs12959006*CC & Reference & Reference \\
\hline \multirow[t]{2}{*}{$N=194$} & rs12959006*CT & $-6.55 \times 10^{-5}(-0.15$ to 0.15$)$ & 0.99 \\
\hline & rs12959006*TT & $-9.99 \times 10^{-2}(-0.53$ to 0.28$)$ & 0.63 \\
\hline
\end{tabular}

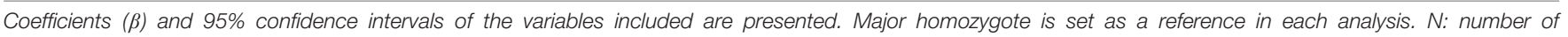
patients in each group. 
TABLE 2 | Poisson regression models for number of relapses at 5 years after MS onset.

\begin{tabular}{|c|c|c|c|}
\hline & Variables included & $\beta(95 \% \mathrm{Cl})$ & $p$ \\
\hline MS overall & rs $12959006^{\star} \mathrm{CC}$ & Reference & Reference \\
\hline \multirow[t]{6}{*}{$N=224$} & rs12959006*CT & $0.08(-0.06$ to 0.21$)$ & 0.27 \\
\hline & rs12959006*TT & $0.36(0.08-0.63)$ & 0.009 \\
\hline & Anti-HHV-6 IgG antibodies & $0.01(0.01-0.02)$ & $3.7 \times 10^{-8}$ \\
\hline & MS duration until antibody measurement & $0.02(0.00-0.03)$ & 0.009 \\
\hline & DMT use & $0.25(0.02-0.48)$ & 0.04 \\
\hline & Onset of MS before 2002 & $0.03(-0.11$ to 0.16$)$ & 0.68 \\
\hline Males MS & rs $12959006^{*} \mathrm{CC}$ & Reference & Reference \\
\hline \multirow[t]{6}{*}{$N=77$} & rs12959006*CT & $0.26(0.03-0.48)$ & 0.02 \\
\hline & rs12959006*TT & $0.74(0.36-1.09)$ & $7 \times 10^{-5}$ \\
\hline & Anti-HHV-6 IgG antibodies & $0.01(0.00-0.02)$ & 0.02 \\
\hline & MS duration until antibody measurement & $0.01(-0.01$ to 0.03$)$ & 0.44 \\
\hline & DMT use & $0.63(0.20-1.07)$ & 0.004 \\
\hline & Onset of MS before 2002 & $-0.15(-0.40$ to 0.09$)$ & 0.23 \\
\hline Females MS & rs12959006*CC & Reference & Reference \\
\hline \multirow[t]{6}{*}{$N=147$} & rs12959006*CT & $-0.03(-0.21$ to 0.15$)$ & 0.73 \\
\hline & rs12959006*TT & $0.06(-0.43$ to 0.49$)$ & 0.80 \\
\hline & Anti-HHV-6 IgG antibodies & $0.02(0.01-0.02)$ & $1.4 \times 10^{-6}$ \\
\hline & MS duration until antibody measurement & $0.03(0.01-0.05)$ & 0.01 \\
\hline & DMT use & $0.12(-0.14$ to 0.39$)$ & 0.37 \\
\hline & Onset of MS before 2002 & $0.09(-0.07$ to 0.26$)$ & 0.29 \\
\hline
\end{tabular}

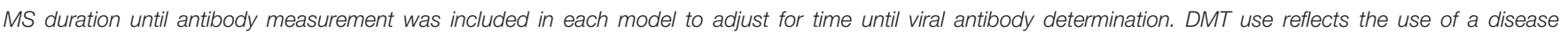

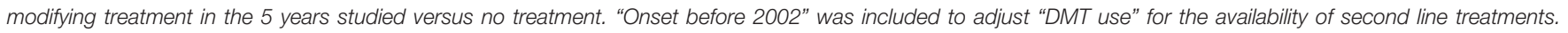

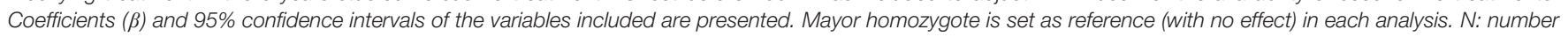
of patients in each group.

with Con A and study of CD69 expression in $\mathrm{CD}^{+}$and $\mathrm{CD}^{-}$cells. Patients were stratified by sex and genotype at rs12959006, and the percentage of positive cells and MFI of CD69 were studied. Due to the low frequency of the rs12959006*TT genotype, only the heterozygote and major homozygote were analyzed. A trend for a lower expression of CD69 in male patients carrying rs12959006*T was observed, although it was not statistically significant (female CT vs. male CT $p=0.09$; Figure 1).

\section{DISCUSSION}

Our study aims at analyzing the putative associations of the MBP polymorphism rs12959006 with MS. A previous study reported associations of this polymorphism with conversion to MS and worse prognosis in these patients (6). The authors studied an Australian cohort of 127 patients with a first demyelinating event compatible with MS. Patients were followed for 5 years. Only 68 patients developed confirmed MS within the duration of the study and they experienced a total of 152 relapses versus the 1292 relapses registered in our study group of 291 MS-confirmed patients. Our analysis benefits from higher statistical power. We describe an association between rs12959006 and risk of a higher number of relapses at 2 and 5 years after MS onset. Interestingly, we observe this association only in male MS patients. MS presents sex differences, being more prevalent in females but more severe in males $(11,12)$.
Recent studies have pointed to other genetic polymorphisms that affect MS only in male patients $(13,14)$ that could explain part of the difference in prevalence and course of MS between sexes.

We observe a trend for a lower expression of CD69 in activated $\mathrm{CD}^{+}$cells in male patients carrying rs $12959006^{*} \mathrm{~T}$ allele. Several studies associate the blocking or lower expression of CD69 in $\mathrm{T}$ cells with an exacerbation of autoimmune diseases $(15,16)$ and a higher induction of pro-inflammatory Th17 cells in mice (17). Moreover, CD69 ${ }^{+} \mathrm{T}$ regulatory cells have been reported to have higher immunosuppressive potential in the mouse model of colitis (18). A lower expression of CD69 in T cells could be related to a defective control of inflammation and a worse evolution of autoimmune diseases. In the light of previous knowledge, it would be interesting to replicate the observed trend in $r s 12959006^{*}$ CT patients and to extend it to the low-frequency rs12959006*TT genotype.

We observe that levels of anti-HHV6 IgG antibodies are positively associated with number of relapses. Other studies $(19,20)$ have previously described associations between high or increasing anti-HHV-6 antibody titers and risk of relapse, and even pointed to a possible predictive value of anti-HHV-6 IgG and IgM levels (19). An elevated antibody titer could suggest a stronger immunological response to HHV-6 infection. Previous studies compared the molecular structure of MBP and HHV-6 proteins. MBP and peptide $\mathrm{U} 24$ of the $\mathrm{HHV}-6$ viral particle share an identical sequence of 6 aminoacids (MBP residues 96-102, HHV-6 U24 residues 4-10), pointing at a possible contribution of 
A

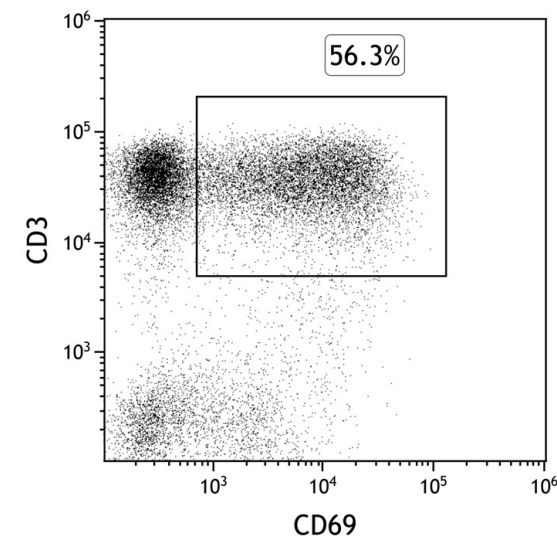

C

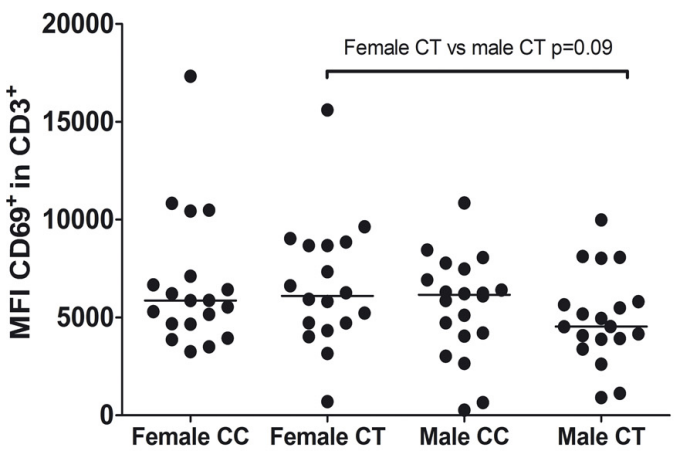

B

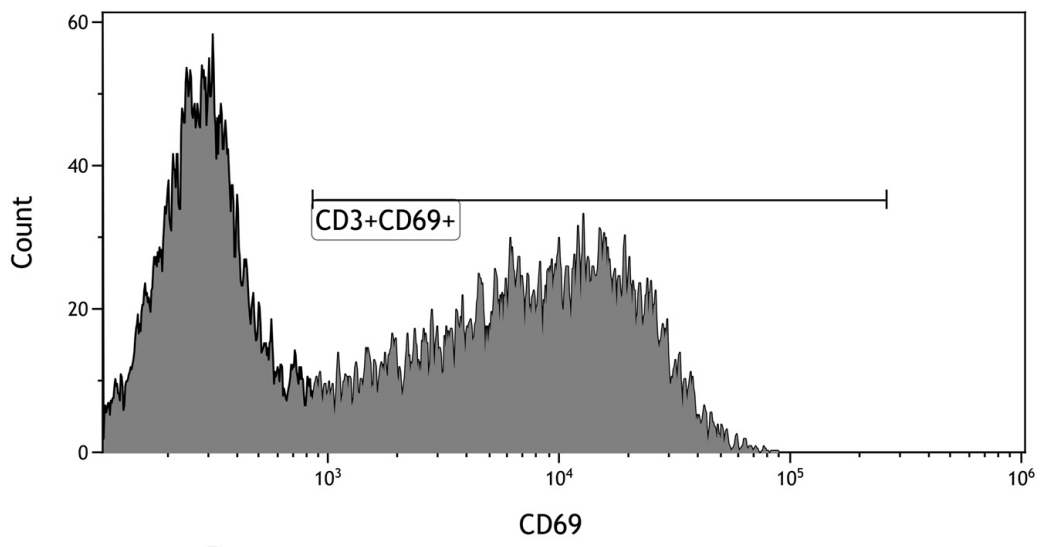

D

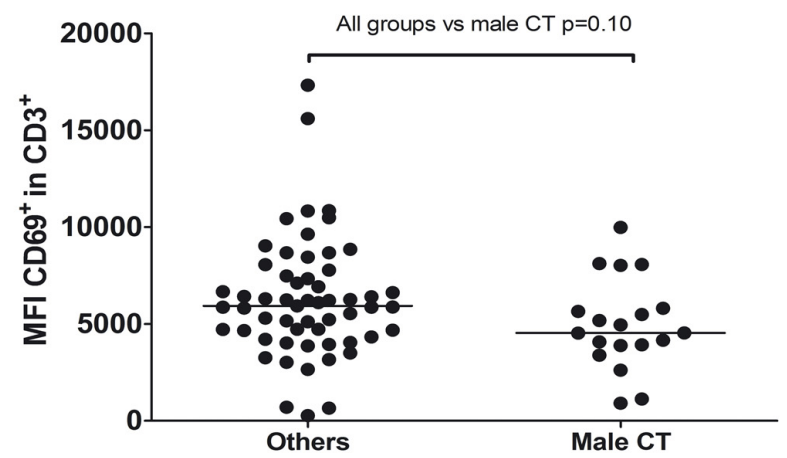

FIGURE 1 | Expression of CD69 in $\mathrm{CD}^{+}$cells measured as MFI according to sex and genotype at rs12959006. (A) Dot plot showing the selection of $\mathrm{CD}^{+} \mathrm{CD}^{+} 9^{+}$ lymphocytes. (B) Histogram showing the selection of $\mathrm{CD} 3^{+} \mathrm{CD} 69^{+}$population for MFI measurement. (C,D) Statistical comparison of $\mathrm{CD} 69$ expression in $\mathrm{CD} 3^{+}$ cells, measured as median fluorescence intensity (MFI), between the four groups studied (C) and all groups vs. male CT (D). Median of each group is represented.

viral infections to MS risk through molecular mimicry. A classic study (3) showed that T-cells cross-reactive to both MBP and HHV-6 were more abundant in MS patients than in healthy controls, an observation that was replicated in a more recent study in Chinese population (21). These studies point to the idea that the joint effect of HHV-6 infection and its molecular mimicry with MBP could be important in MS pathogenesis.

For future research, it would be interesting to replicate these observations in a group with baseline anti-HHV-6 IgG antibody measurements and in the context of a prospective study to determine the usefulness of rs12959006 in clinical practice.

Our study, and those from others, consolidates the contribution of rs12959006, within the $M B P$ gene, as a marker to predict MS activity. Furthermore, according to our results, there seems to be an influence of both the genetic polymorphism in $M B P$ and HHV-6 infection in predicting a higher relapse rate that is exclusive to male MS patients.

\section{DATA AVAILABILITY STATEMENT}

All datasets generated for this study are included in the article/supplementary material.

\section{ETHICS STATEMENT}

The studies involving human participants were reviewed and approved by the Comité Ético de Investigación Clínica, Hospital Clínico San Carlos, Madrid (Spain). The patients/participants provided their written informed consent to participate in this study.

\section{AUTHOR CONTRIBUTIONS}

LE-P, TA-J, IR-M, PL-C, MG-M, MD-M, SP-P, and RD-C performed the experiments and collected the clinical data. LE-P, MC, EU and RÁ-L designed the experiments, analyzed the data, and drafted the manuscript.

\section{FUNDING}

This work was supported by the projects PI15/00821 and PI16/01259, integrated in the Plan National de I+D+I, AES 2013-2016; funded by the ISCIII and co-funded by the European Regional Development Fund (ERDF) "A way to make Europe"; "Fundación Ramón Areces" (CIVP18A3860) 
and "Fundación LAIR". LE-P and MG-M are recipients of a contract from "REEM: Red Española de Esclerosis Múltiple” (RETICS-REEM RD16/0015/0013; www.reem.es). SP$\mathrm{P}$ holds a research contract from the program "Formación de Profesorado Universitario" - Ministerio de Educación, Cultura y Deporte" (FPU16/00969).

\section{REFERENCES}

1. Reich DS, Lucchinetti CF, Calabresi PA. Multiple sclerosis. $N$ Engl J Med. (2018) 378:169-80.

2. Rangachari M, Kuchroo VK. Using EAE to better understand principles of immune function and autoimmune pathology. J Autoimmun. (2013) 45:31-9. doi: 10.1016/j.jaut.2013.06.008

3. Tejada-Simon MV, Zang YC, Hong J, Rivera VM, Zhang JZ. Cross-reactivity with myelin basic protein and human herpesvirus- 6 in multiple sclerosis. Ann Neurol. (2003) 53:189-97.

4. Axisa PP, Hafler DA. Multiple sclerosis: genetics, biomarkers, treatments. Curr Opin Neurol. (2015) 29:345-53.

5. Baranzini SE, Oksenberg JR. The genetics of multiple sclerosis: from 0 to 200 in 50 years. Trends Genet. (2017) 33:960-70. doi: 10.1016/j.tig.2017. 09.004

6. Zhou Y, Simpson S Jr., Charlesworth JC, van der Mei I, Lucas RM, Ponsonby $\mathrm{AL}$, et al. Variation within MBP gene predicts disease course in multiple sclerosis. Brain Behav. (2017) 7:e00670. doi: 10.1002/brb3.670

7. McDonald WI, Compston A, Edan G, Goodkin D, Hartung HP, Lublin FD, et al. Recommended diagnostic criteria for multiple sclerosis: guidelines from the International Panel on the diagnosis of multiple sclerosis. Ann Neurol. (2001) 50:121-7.

8. Polman CH, Reingold SC, Banwell B, Clanet M, Cohen JA, Filippi M, et al. Diagnostic criteria for multiple sclerosis: 2010 revisions to the McDonald criteria. Ann Neurol. (2011) 69:292-302. doi: 10.1002/ana.22366

9. Polman CH, Reingold SC, Edan G, Filippi M, Hartung HP, Kappos L, et al. Diagnostic criteria for multiple sclerosis: 2005 revisions to the "McDonald Criteria”. Ann Neurol. (2005) 58:840-6.

10. Poser CM, Paty DW, Scheinberg L, McDonald WI, Davis FA, Ebers GC, et al. New diagnostic criteria for multiple sclerosis: guidelines for research protocols. Ann Neurol. (1983) 13:227-31.

11. Ysrraelit MC, Correale J. Impact of sex hormones on immune function and multiple sclerosis development. Immunology. (2018) 156:9-22. doi: 10.1111/ imm.13004

12. Golden LC, Voskuhl R. The importance of studying sex differences in disease: the example of multiple sclerosis. J Neurosci Res. (2017) 95:633-43. doi: 10. 1002/jnr.23955

13. Han Z, Qu J, Zhao J, Zou X. Genetic variant rs755622 regulates expression of the multiple sclerosis severity modifier D-Dopachrome tautomerase in a sex-specific way. Biomed Res Int. (2018) 2018:8285653. doi: 10.1155/2018/ 8285653

\section{ACKNOWLEDGMENTS}

The authors wish to thank the "Unidad de apoyo metodológico a la investigación" (UAMI) of the Instituto de Investigaciones Sanitarias San Carlos (IdISSC) for their expert assistance in statistical analysis.

14. Benedek G, Meza-Romero R, Jordan K, Zhang Y, Nguyen H, Kent G, et al. MIF and D-DT are potential disease severity modifiers in male MS subjects. Proc Natl Acad Sci USA. (2017) 114:E8421-9. doi: 10.1073/pnas.1712288114

15. Gonzalez-Amaro R, Cortes JR, Sanchez-Madrid F, Martin P. Is CD69 an effective brake to control inflammatory diseases? Trends Mol Med. (2013) 19:625-32. doi: 10.1016/j.molmed.2013.07.006

16. Radstake TR, van Bon L, Broen J, Wenink M, Santegoets K, Deng Y, et al. Increased frequency and compromised function of $\mathrm{T}$ regulatory cells in systemic sclerosis (SSc) is related to a diminished CD69 and TGFbeta expression. PLoS One. (2009) 4:e5981. doi: 10.1371/journal.pone.0005981

17. Martin P, Gomez M, Lamana A, Cruz-Adalia A, Ramirez-Huesca M, Ursa MA, et al. CD69 association with Jak3/Stat5 proteins regulates Th17 cell differentiation. Mol Cell Biol. (2010) 30:4877-89. doi: 10.1128/MCB.00456-10

18. Yu L, Yang F, Zhang F, Guo D, Li L, Wang X, et al. CD69 enhances immunosuppressive function of regulatory T-cells and attenuates colitis by prompting IL-10 production. Cell Death Dis. (2018) 9:905. doi: 10.1038/ s41419-018-0927-9

19. Ortega-Madueno I, Garcia-Montojo M, Dominguez-Mozo MI, GarciaMartinez A, Arias-Leal AM, Casanova I, et al. Anti-human herpesvirus 6A/B IgG correlates with relapses and progression in multiple sclerosis. PLoS One. (2014) 9:e104836. doi: 10.1371/journal.pone.0104836

20. Simpson S Jr., Taylor B, Dwyer DE, Taylor J, Blizzard L, Ponsonby AL, et al. Anti-HHV-6 IgG titer significantly predicts subsequent relapse risk in multiple sclerosis. Mult Scler. (2012) 18:799-806. doi: 10.1177/135245851 1428081

21. Cheng W, Ma Y, Gong F, Hu C, Qian L, Huang Q, et al. Cross-reactivity of autoreactive T cells with MBP and viral antigens in patients with MS. Front Biosci (Landmark Ed). (2012) 17:1648-58. doi: 10.2741/4010

Conflict of Interest: The authors declare that the research was conducted in the absence of any commercial or financial relationships that could be construed as a potential conflict of interest.

Copyright (C) 2020 Espino-Paisán, Agudo-Jiménez, Rosales-Martínez, LópezCotarelo, García-Martínez, Domínguez-Mozo, Pérez-Pérez, Dieli-Crimi, Comabella, Urcelay and Álvarez-Lafuente. This is an open-access article distributed under the terms of the Creative Commons Attribution License (CC BY). The use, distribution or reproduction in other forums is permitted, provided the original author(s) and the copyright owner(s) are credited and that the original publication in this journal is cited, in accordance with accepted academic practice. No use, distribution or reproduction is permitted which does not comply with these terms. 